\title{
Do monitoramento do desempenho ao desempenho do monitoramento: novas oportunidades para a Avaliação na gestão da Vigilância em Saúde
}

From monitoring of performance to the performance of monitoring: new opportunities for evaluation in the management of Health Surveillance

Zulmira Hartz ${ }^{3}$

“... as avaliações devem ter um papel fundamental nos sistemas de mensuração do desempenho, muitas vezes chamados de sistemas de 'monitoramento e avaliação', sendo que, na prática, pouca atenção é dada ao componente de avaliação"1.

In Canada, the concept of monitoring and evaluation (M\&E) is interpreted such that evaluation has a distinct identity from monitoring... It should be noted that this is not always true in other parts of the world where the term MeE may ignore or minimize the concept of evaluation ${ }^{2}$.

\footnotetext{
${ }^{3}$ Instituto de Higiene e Medicina Tropical, Universidade Nova de Lisboa.zhartz@ihmt.unl.pt
} 
Antes de mais nada agradeço aos colegas avaliadores o convite para participar deste forum de discussão sobre a inovadora proposta de monitoramento construida para a gestão da vigilância em saúde de Pernambuco. Entendendo que a proposta, na sua abordagem, ultrapassa a construção dos limites de um "instrumento" de monitorização (como referido no resumo), para se constituir um sistema de monitoramento qualificado como uma intervenção capaz de efetivamente favorecer o desempenho da vigilância e da saúde naquele estado. É nesta perspectiva que gostaria de alinhar este breve comentário, ressaltando as vantagens de que seja ele próprio objeto e projeto de uma política institucional de avaliação da performance.

Assim começaria destacando a importância dada na literatura do desempenho das políticas e programas sociais, à necessidade de se ter idêntica preocupação com o desempenho de sua avaliação, particularmente no campo das intervenções em saúde. De uma forma pragmática pretende-se contribuir para superar as inúmeras discussões e impasses metodológicos sobre medidas e indicadores de performance privilegiados, na comparabilidade dos sistemas locais nacionais de saúde, para focalizar na exigência de se fazer uma gestão integrada e contextualizada do desempenho e da avaliação.

Correndo o risco de me repetir nessas reflexões, que assumo como um a priori, elas se inspiram fortemente na experiência Canadense de institucionalização conduzida por Mayne no inícios dos anos 80 e nas lições aprendidas com os 30 anos deste sistema de Monitoramento e Avaliação (autoria de Robert Lahey) recentemente publicada pelo Banco Mundial ${ }^{1,2}$. O pressuposto de base é de que a maneira como o monitoramento e a avaliação são organizados nos diversos níveis do sistema terá um impacto significativo nos tipos de estudos que são conduzidos e utilizados. Embora considerando-se inevitável que estas duas atividades estejam intimamente relacionadas, há consenso de que é necessário distinguir a natureza estratégica e investigativa da avaliação, do enfoque gerencial e normativo do monitoramento, em sua inserção decisional.

Se as concepções de institucionalização da avaliação das políticas públicas são inúmeras, a construção de uma cultura avaliativa, que faz do uso da avaliação parte integral das operações de uma organização ou sistema social (mainstreaming evaluation), é consensualmente o efeito esperado de sua adequada implementação, tendo como prin- cipal determinante o fortalecimento da capacidade de todos os atores envolvidos, através de processos formais e continuados de aprendizagem, em que erros e falhas, resultantes de inovações ou experimentos com vistas a melhores resultados, não são punidos e sim computados como lições aprendidas ${ }^{3,4}$. Esta convicção partilhada entre Mayne e membros do Grupo de Estudos de Gestão e Avaliação em Saúde do Instituto de Medicina Integral Prof. Fernando Figueira (Geas/Imip), também presentes na autoria dessa promissora iniciativa aqui apresentada, me permite acreditar que estamos realmente diante de uma oportunidade ímpar para implantar um monitoramento de gestão da vigilância como catalisador de um processo institucional e estadual, em que a avaliação seja devidamente valorizada e reconhecida como a melhor alternativa de se obter informações sobre a performance de um sistema de saúde, se justificando como prática essencial de racionalização e das decisões em matéria de alocação de recursos. Para tal, precisará demonstrar, também como (meta) avaliando, que efeitos ou impactos podem ser imputados, como contribuição de suas ações ou práticas avaliativas, e a que custo.

Por fim gostaria de destacar que, embora tenha me limitado a falar um pouco da experiência canadense, uma rica literatura da avaliação da performance na união europeia tem assumido como de incontornável importância a estabilidade, ainda que provisória, dada pela avaliação pois ... 'truth' will be based on what is reasonable to conclude when the array of evidence is weighed ${ }^{5}$.

\section{Referências}

1. Mayne J. Challenges and Lessons in Implementing Results-Based Management. Evaluation 2007; 13(1):87109.

2. Lahey R. The Canadian M\&E System: Lessons Learned from 30 years of Development. Washington: Word Bank ECD Working Paper Series, n²3; 2010.

3. Mayne J. Building an evaluative culture: the key of effective evaluation and results management. CJPE 2010; 24(2):1-30.

4. Felisberto E, Alves CKA, Bezerra LCA. (). Institucionalização da Avaliação. In: Samico I, Felisberto E, Figueiró AC, Frias PG, organizadores. Avaliação em Saúde. Bases Conceituais e Operacionais. Rio de Janeiro: Medbook; 2010. p. 143-160.

5. Saunders M. Capturing effects of interventions, policies and programmes in the European Context: A social practice perspective. Evaluation 2011; 17(1):89-102. 